\title{
The Impact of Sonication on the Surface Quality of Single-Walled Carbon Nanotubes
}

\author{
BYUMSEOK KOH, WEI CHENG
}

Department of Pharmaceutical Sciences, University of Michigan, Ann Arbor, Michigan 48109

\author{
Received 16 December 2014; revised 14 March 2015; accepted 10 April 2015
}

Published online 27 May 2015 in Wiley Online Library (wileyonlinelibrary.com). DOI 10.1002/jps.24483

\begin{abstract}
Sonication process is regularly adopted for dispersing single-walled carbon nanotubes (SWCNTs) in an aqueous medium. This can be achieved by either covalent functionalization of SWCNTs with strong acid or by noncovalent functionalization using dispersants that adsorb onto the surface of SWCNTs during dispersion. Because the dispersion process is usually performed using sonication, unintentional free radical formation during sonication process may induce covalent modification of SWCNT surface. Herein, we have systematically investigated the status of SWCNT surface modification under various sonication conditions using Raman spectroscopy. Comparing $I_{\mathrm{D}} / I_{\mathrm{G}}$ (Raman intensities between D and G bands) ratio of SWCNTs under various sonication conditions suggests that typical sonication conditions (1-6 h bath sonication with sonication power between 3 and $80 \mathrm{~W}$ ) in aqueous media do not induce covalent modification of SWCNT surface. In addition, we confirm that SWCNT dispersion with single-stranded DNA (ssDNA) involves noncovalent adsorption of ssDNA onto the surface of SWCNTs, but not covalent linkage between ssDNA and SWCNT surface. (C) 2015 Wiley Periodicals, Inc. and the American Pharmacists Association J Pharm Sci 104:2594-2599, 2015
\end{abstract}

Keywords: single-walled carbon nanotubes; sonication; surface modification

\section{INTRODUCTION}

There is an ever-increasing interest in the use of single-walled carbon nanotubes (SWCNTs) for pharmaceutical applications, either as potential drug or gene delivery tools ${ }^{1-4}$ or as tissue scaffolds. ${ }^{5,6}$ Many of these applications are owing to their unique mechanical, electrical, and thermal properties. SWCNTs typically exist as water-insoluble bundles. ${ }^{78}$ For various pharmaceutical applications, it is almost inevitable that one needs to disperse SWCNT bundles into an aqueous medium in order to be biologically compatible. Aggregated instead of dispersed SWCNTs have been shown to induce toxicity in vivo. ${ }^{9}$ Thus, there is a need for methods to disperse and stabilize SWCNTs in aqueous media.

Two types of methods have been developed in the literature for the dispersion of SWCNTs in aqueous media. The first type involves the treatment of SWCNTs with strongly oxidative acids, ${ }^{10-12}$ which oxidize the surface of SWCNTs and give rise to hydrophilic groups that afford the dispersion of SWCNTs in aqueous media. ${ }^{13}$ Although efficient, this strong acid treatment and the resulting highly modified surface of SWCNTs are not usually compatible with downstream applications. ${ }^{14,15}$ Modification of SWCNT surface through noncovalent interactions is thus desired to achieve dispersion in aqueous media. ${ }^{16,17}$ This method involves the use of a dispersant molecule, which is typically amphiphilic in nature. ${ }^{18-21}$ Sonication $(3-15 \mathrm{~W}, 1 \mathrm{~h})$ of SWCNTs with dispersant in a $\mathrm{pH} \sim 6$ deionized water $\left(\mathrm{ddH}_{2} \mathrm{O}\right)$ system induces adsorption of dispersant onto the surface of SWCNTs via $\pi$-stacking or Van der Waals interactions. ${ }^{18-20}$ The hydrophilic groups in the dispersant molecules thus afford the dispersion of SWCNTs.

Correspondence to: Wei Cheng (Telephone: +734-763-3709; Fax: +734-6156162; E-mail: chengwe@umich.edu)

This article contains supplementary material available from the authors upon request or via the Internet at http://wileylibrary.com.

Journal of Pharmaceutical Sciences, Vol. 104, 2594-2599 (2015)

(C) 2015 Wiley Periodicals, Inc. and the American Pharmacists Association
Although this noncovalent functionalization of SWCNT surface does not involve any covalent modification of SWCNT surface, ${ }^{22-24}$ the impact of sonication procedure itself on SWCNT surface is less certain. Sonication in an aqueous solution is known to generate free radicals such as hydroxyl radical $\left(\mathrm{OH}\right.$.) and the super-oxide ion $\left(\mathrm{O}_{2}{ }^{-}\right){ }^{25,26}$ These highly reactive species may chemically modify the $\mathrm{sp}^{2}$ carbons on the surface of SWCNTs, and further mediate the covalent attachment of dispersant molecules on SWCNT surface. Indeed, Hines ${ }^{27}$ reported that covalent linkage between SWCNT and short singlestranded DNA (ssDNA) may occur after sonication process in water. Although sonication procedure is widely adopted in SWCNT community for their dispersion in aqueous media, the extent of covalent modification of SWCNT surface through sonication has not been quantified or reported. For pharmaceutical applications where the molecules of interest are associated with SWCNT surface through noncovalent adsorption for delivery into biological milieu, the covalent attachment of these molecules will unavoidably compromise the release of these molecules. Therefore, in this paper, we have carried out a systematic study on the effect of sonication on SWCNT surface modification, the results of which should be useful for the application of SWCNTs as drug or gene delivery tools.

\section{EXPERIMENTAL}

\section{Preparation of Individually Dispersed SWCNTs}

One milligram of as-prepared SWCNTs soot produced from arc discharge method (AD SWCNTs; Helix Materials Solution, Richardson, Texas), or chemical vapor deposition (CVD; SES Research, Houston, Texas), or high-pressure carbon monoxide process (HiPCO, Super purified; Unidym, Sunnyvale, California) was added to $1 \mathrm{~mL}$ distilled water and $\mathrm{ddH}_{2} \mathrm{O}(\mathrm{Syn}-$ ergy UV, Millipore, Massachusetts) together with $1 \mathrm{mg}$ of a ssDNA oligo, $(\mathrm{dT})_{30}$ (IDT, Coralville, Iowa) in a 1.5-mL centrifuge 
tube. The mixture was then subjected to bath sonication under various conditions as indicated throughout the paper. The sonication was performed either using a tabletop sonicator (Table Ultra Sonic Cleaner, FS-20H; Thermo Fisher Scientific, Waltham, Massachusetts) with an output power $\sim 3 \mathrm{~W}$ for various amount of time as indicated, or using a variable-power sonication system (Ultrasonic Processor, S-4000; Misonix, Farmingdale, New York) for $1 \mathrm{~h}$ at various output power settings $(10,20,40$, and $80 \mathrm{~W})$. For sonication in the latter system, $15 \mathrm{~s}$ of sonication was followed by $5 \mathrm{~s}$ idleness for each step, and the step was repeated until the total on-time reached $1 \mathrm{~h}$. For both sonication apparatus, ice was constantly added to the water bath surrounding the centrifuge tube to prevent overheating throughout the entire sonication process. Dispersed SWCNTs were centrifuged at $17,000 \mathrm{~g}$ for $1 \mathrm{~h}$ at room temperature (Sorvall Legend Micro 17; Thermo Fisher Scientific). Supernatants were collected, and the fraction of individually dispersed SWCNTs was estimated by recording absorbance at $1023 \mathrm{~nm}$ using a UV/Vis spectrophotometer (UV-1800; Shimadzu, Kyoto, Japan), as we described previously. ${ }^{20}$

\section{Raman Spectroscopy of SWCNTs}

Ten microliter of dispersed SWCNT samples in $\mathrm{ddH}_{2} \mathrm{O}$ was placed onto aluminum foil on a glass slide $\left(75 \times 25 \times 1 \mathrm{~mm}^{3}\right.$; Thermo Fisher Scientific) and analyzed using two different wavelengths of laser (514 and $633 \mathrm{~nm}$, inVia Raman microscope; Renishaw Inc., Hoffman Estates, Illinois). The microscope was operated at $1 \%$ laser power with a $100 \times$ objective lens (BX41; Olympus, Tokyo, Japan) and $30 \mathrm{~s}$ exposure time. For each sample, the Raman peak intensities for D-band $\left(I_{\mathrm{D}}\right.$, $\left.\sim 1350 \mathrm{~cm}^{-1}\right)$ and G-band $\left(I_{\mathrm{G}}, \sim 1590 \mathrm{~cm}^{-1}\right)$ were taken directly from the spectra, and their ratios were calculated as reported. ${ }^{28-32}$ Carboxylated SWCNTs (SWCNT-COOH, or P3-
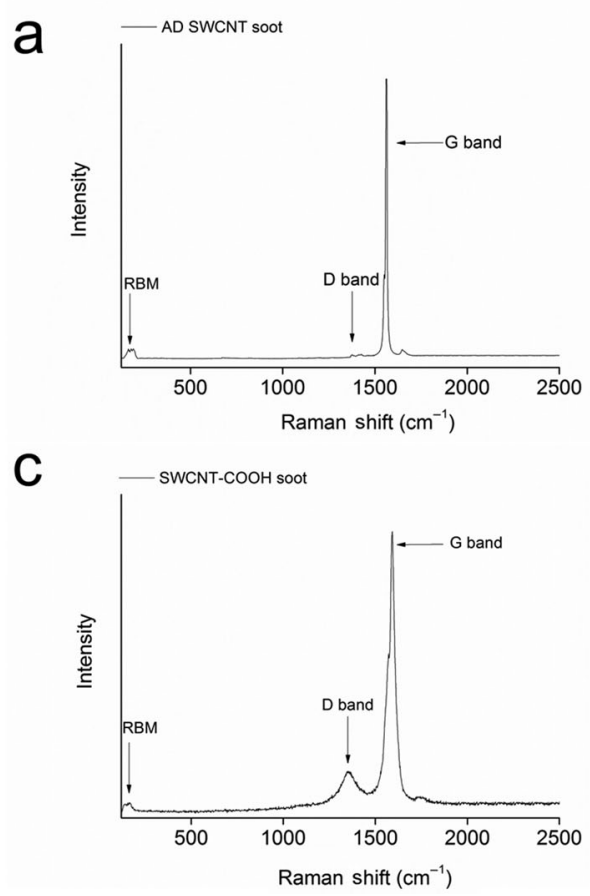

SWNT from Carbon Solutions Inc., Riverside, California) and amide-functionalized SWCNTs (SWCNT-CONH 2 , or P9-SWNT from Carbon Solutions Inc.) were used and measured as the original soot without dispersion.

Dispersion of SWCNTs in the Presence of Ascorbic Acid or Trolox

One milligram of AD, CVD, or HiPCO SWCNTs was dispersed in $1 \mathrm{~mL}$ of ascorbic acid or trolox (antioxidant) ${ }^{33,34}$ aqueous solution in the presence of $1 \mathrm{mg}$ of $(\mathrm{dT})_{30}$ through sonication using the tabletop sonicator at $\sim 3 \mathrm{~W}$ for various amount of time as indicated. The concentration of ascorbic acid or trolox was $0.5 \mathrm{mg} / \mathrm{mL}$ unless otherwise noted. To reduce the concentration of $\mathrm{O}_{2}$ in the solution that may facilitate the generation of radicals, we also conducted sonication after argon purging. This was performed by placing the $1.5-\mathrm{mL}$ centrifuge tube with sample mixtures in a vacuum desiccator. With the tube cap open, we pulled with house vacuum for $1 \mathrm{~h}$. Argon stream was then applied for $1 \mathrm{~min}$. The cap of the tube was then closed and sealed with Parafilm (Bemis NA, Neenah, Wisconsin) before sonication. All reagents were from Sigma-Aldrich (Saint Louis, Missouri) unless specified.

\section{RESULTS AND DISCUSSION}

\section{Raman Spectroscopy of SWCNTs}

To test whether sonication process induces covalent modification of SWCNTs, Raman spectra ${ }^{28-32}$ of AD, CVD, and HiPCO SWCNTs after dispersion with $(\mathrm{dT})_{30}(1 \mathrm{~h}$ at $3 \mathrm{~W})$ were analyzed and compared with SWCNTs before dispersion (SWCNT soot). Typical Raman spectra of AD SWCNT before and after dispersion were shown in Figures $1 \mathrm{a}$ and 1b, respectively. For comparison, we also collected the Raman spectra for SWCNT soot that were functionalized by the manufacturer, as shown in Figure 1c
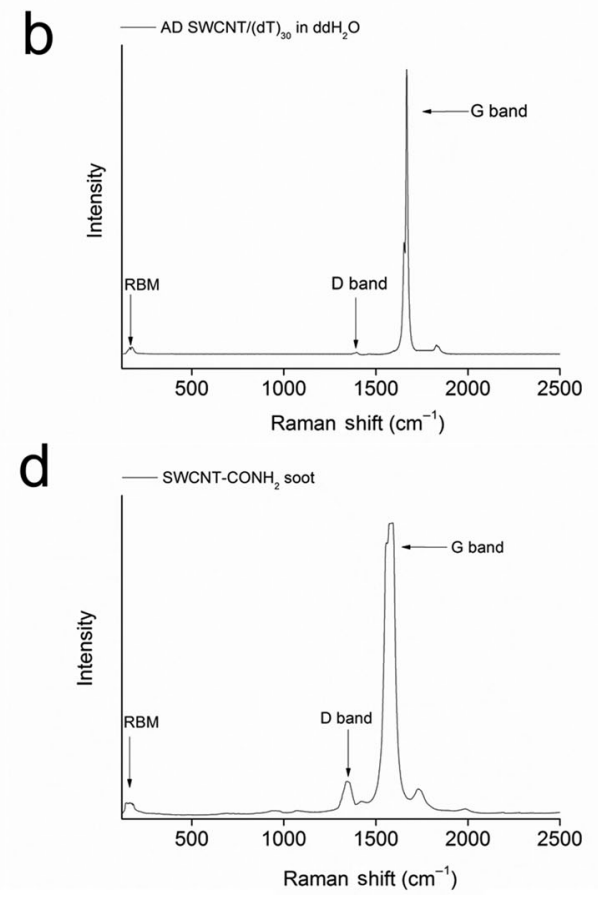

Figure 1. Raman spectra recorded using $514 \mathrm{~nm}$ laser for (a) AD SWCNT soot, (b) AD SWCNT/(dT) $)_{30}$ dispersed in water, (c) SWCNT-COOH soot, and (d) SWCNT-CONH 2 soot. 


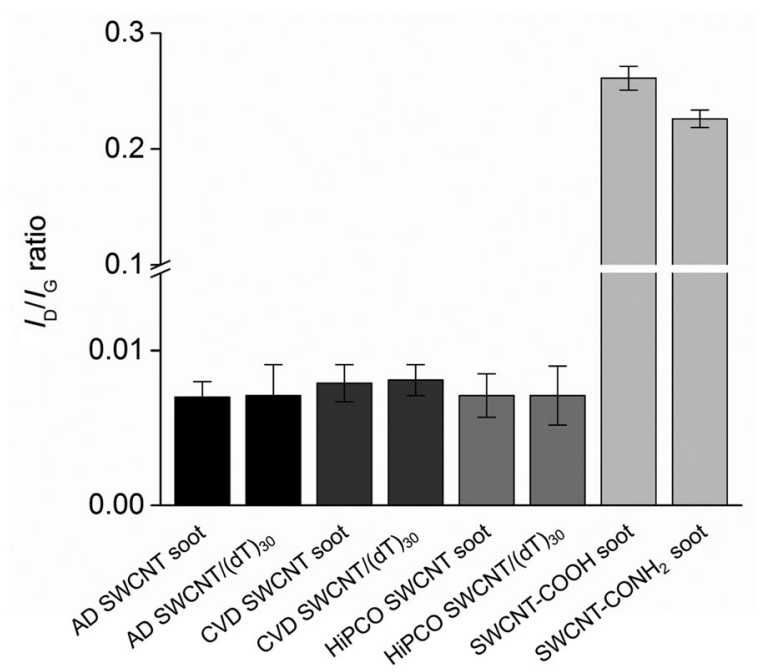

Figure 2. $I_{\mathrm{D}} / I_{\mathrm{G}}$ ratio of different types of SWCNTs. Error bars represent SD from three independent repeats of the same experiments.

for SWCNT-COOH and Figure 1d for SWCNT-CONH $\mathrm{H}_{2}$. The D-band in SWCNT Raman spectra results from defect along SWCNT and covalently functionalized SWCNTs show a clear D-band signal around $1350 \mathrm{~cm}^{-1} \cdot{ }^{30}$ At a given laser wavelength, this ratio is directly proportional to the density of defects on SWCNT surface. ${ }^{29}$ As a result, the quality of an SWCNT sample is evaluated by comparing the Raman intensities between $\mathrm{D}$ and $\mathrm{G}$ bands, characterized by the $I_{\mathrm{D}} / I_{\mathrm{G}}$ ratio. ${ }^{30}$ For highquality samples, the $I_{\mathrm{D}} / I_{\mathrm{G}}$ ratio is often below 0.01 . As shown in Figure 1, the D-bands for AD SWCNT soot and AD SWCNT dispersed in water with $(\mathrm{dT})_{30}\left(\mathrm{SWCNT} /(\mathrm{dT})_{30}\right)$ are both very subtle. In contrast, both SWCNT-COOH and SWCNT-CONH show apparent D-bands around $1350 \mathrm{~cm}^{-1}$. The $I_{\mathrm{D}} / I_{\mathrm{G}}$ ratio for $\mathrm{AD}$ SWCNT soot was $0.007 \pm 0.001$ (mean $\pm \mathrm{SD}$ throughout), consistent with being a high-quality SWCNT sample without many defects. The $I_{\mathrm{D}} / I_{\mathrm{G}}$ ratio for $\mathrm{AD} \mathrm{SWCNT} /(\mathrm{dT})_{30}$ was $0.007 \pm 0.002$, which is identical to that of AD SWCNT soot within error, indicating that $1 \mathrm{~h}$ sonication using a tabletop sonicator $(\sim 3 \mathrm{~W})$ does not introduce any significant covalent modification of AD SWCNTs. In contrast, SWCNT-COOH and SWCNT-CONH $H_{2}$ showed average $I_{\mathrm{D}} / I_{\mathrm{G}}$ ratio of $0.261 \pm 0.010$ and $0.226 \pm 0.008$, respectively, indicating that both of these functionalized SWCNTs contain significant covalent modifications on their surface. Figure 2 shows the values of $I_{\mathrm{D}} / I_{\mathrm{G}}$ ratio for the different SWCNT samples that we have prepared via sonication for $1 \mathrm{~h}$ at $\sim 3 \mathrm{~W}$ in comparison with various SWCNT soots. For all these samples except the two made-tofunctionalized SWCNTs, they all have an $I_{\mathrm{D}} / I_{\mathrm{G}}$ ratio that is less than 0.01 . There are no changes in $I_{\mathrm{D}} / I_{\mathrm{G}}$ ratio within error before and after sonication for $1 \mathrm{~h}$ at $\sim 3 \mathrm{~W}$.

\section{Effect of Sonication Time and Power on the Quality of SWCNT Dispersion}

We have shown previously that a long sonication time or high sonication power during the dispersion of SWCNT leads to a significant reduction in SWCNT length. ${ }^{20}$ This breakage of SWCNT as a result of ultrasonic processing may lead to covalent modification of SWCNTs, especially at the broken ends of individual SWCNTs. To investigate the effect of sonication time and power on the quality of dispersed SWCNTs, we measured Raman spectra for samples dispersed under various conditions, and calculated the $I_{\mathrm{D}} / I_{\mathrm{G}}$ ratio for each sample for the comparison. As shown in Figure 3a for AD, CVD, and HiPCO SWCNTs dispersed in the presence of $(\mathrm{dT})_{30}$ at various powers, there is a small but measureable trend of increase in $I_{\mathrm{D}} / I_{\mathrm{G}}$ ratio with increasing sonication power. This small trend of increase of $I_{\mathrm{D}} / I_{\mathrm{G}}$ ratio to $0.008,0.009$, and 0.007 for $\mathrm{AD}, \mathrm{CVD}$, and HiPCO SWCNTs, respectively, as the power was increased from 3 to $80 \mathrm{~W}$. Similarly, as shown in Figure 3b for AD, CVD, and HiPCO SWCNTs dispersed in the presence of $(\mathrm{dT})_{30}$ in a tabletop sonicator $(\sim 3 \mathrm{~W})$ but with various lengths of sonication time, there is also a small but measureable trend of increase in $I_{\mathrm{D}} / I_{\mathrm{G}}$ ratio with increasing sonication time. This small trend of $I_{\mathrm{D}} / I_{\mathrm{G}}$ ratio to $0.008,0.009$, and 0.007 for AD, CVD, and HiPCO SWCNTs, respectively, as the time was increased from $30 \mathrm{~min}$ to $12 \mathrm{~h}$. Regardless, all these $I_{\mathrm{D}} / I_{\mathrm{G}}$ ratios remained below 0.01 throughout the different sonication procedures. These data suggest that although higher power or longer time of sonication will lead to more covalent modifications of SWCNTs (oxidation of $\mathrm{sp}^{2}$ carbon to $\mathrm{sp}^{3}$ for example), which likely occur around the broken ends of individual SWCNTs, the overall effect is very small and the dispersed SWCNTs remain in high quality.

\section{Effects of Antioxidants on SWCNT Dispersion}

Although extensive studies on SWCNT dispersion in the presence of ssDNA suggest that ssDNA [including $(\mathrm{dT})_{30}$ ] adsorbs
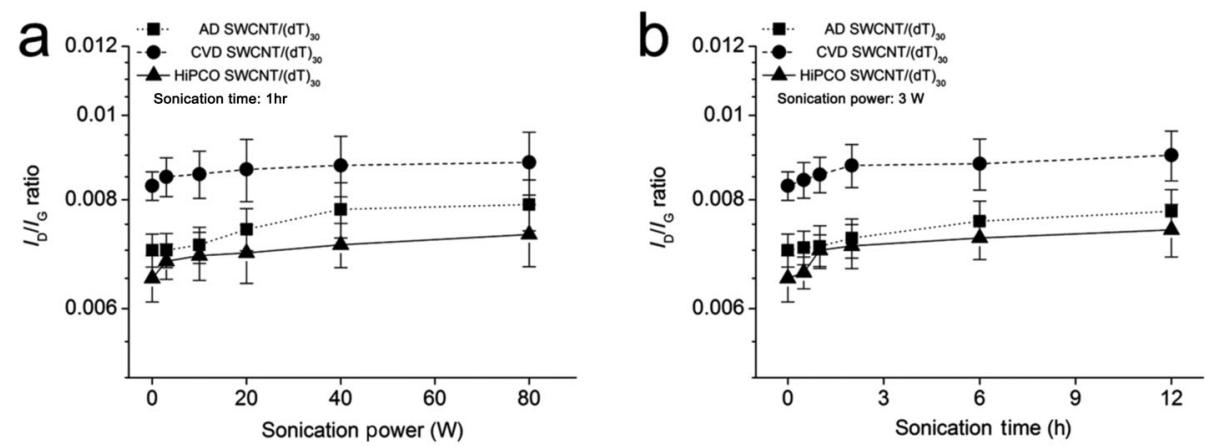

Figure 3. The impact of sonication power (a) and sonication time (b) on the quality of dispersed SWCNTs. The $I_{\mathrm{D}} / I_{\mathrm{G}}$ ratios for SWCNT samples dispersed under various conditions are shown for $\mathrm{AD}, \mathrm{CVD}$, and HiPCO SWCNT/(dT) 30 , as a function of sonication time in (a) or a function of sonication power in (b). Error bars represent SD from three independent repeats of the same experiments. 

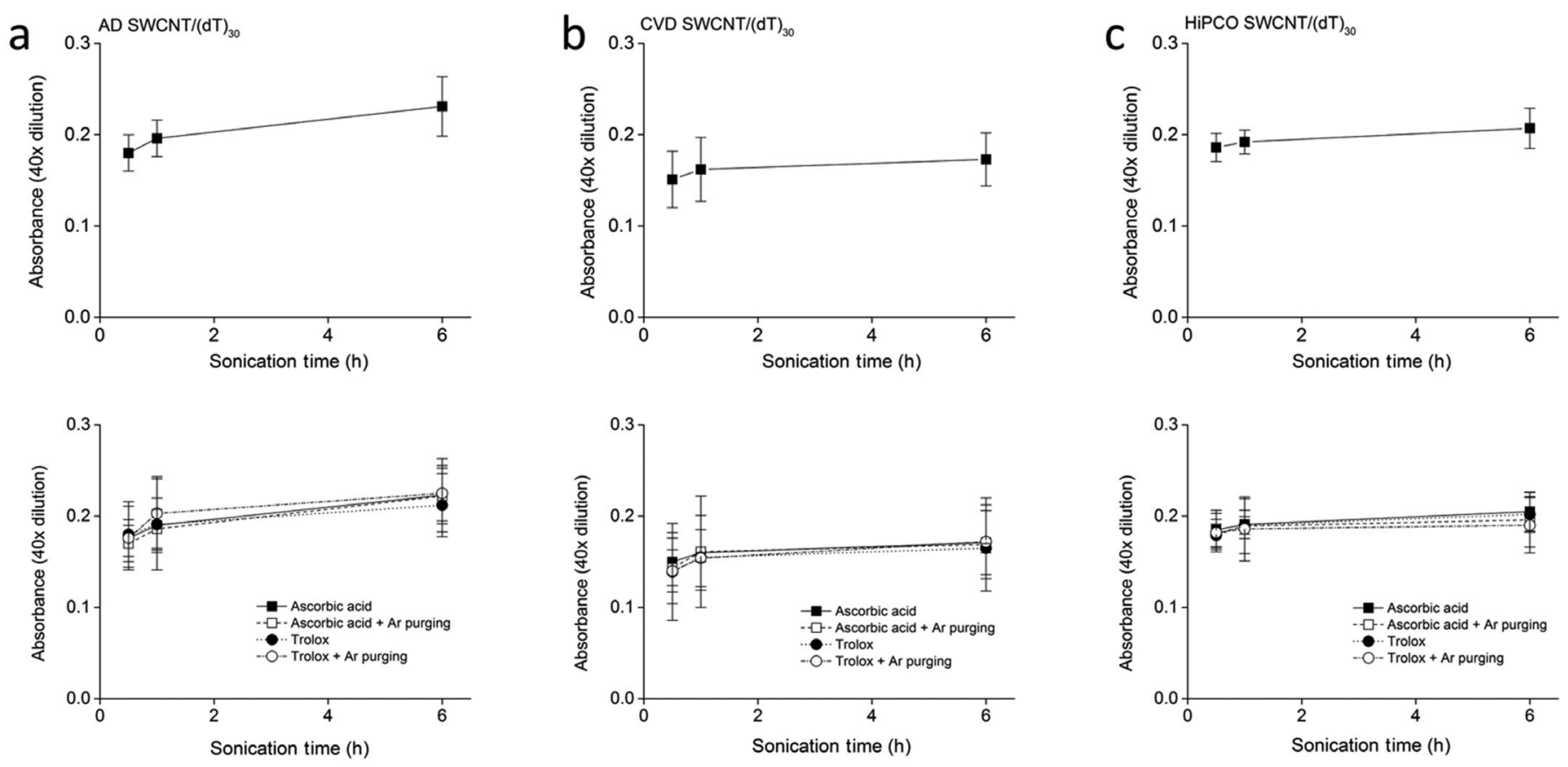

Figure 4. The dispersion of SWCNT samples in the absence (a) or presence of various antioxidants (b). The amount of dispersed SWNCTs was monitored by absorbance at $1023 \mathrm{~nm}$. Using a sonication power of $\sim 3 \mathrm{~W}$, the amount of SWCNTs dispersed into an aqueous solution as a function of sonication time was shown in (a), (b), and (c) for AD, CVD, and HiPCO SWCNT/ $(\mathrm{dT})_{30}$, respectively. The top panels are for dispersions in $\mathrm{ddH}_{2} \mathrm{O}$ without antioxidants, and the bottom panels are for dispersions in $\mathrm{ddH}_{2} \mathrm{O}$ in the presence of ascorbic acid or trolox, with or without argon purging. Error bars represent SD from three independent repeats of the same experiments.

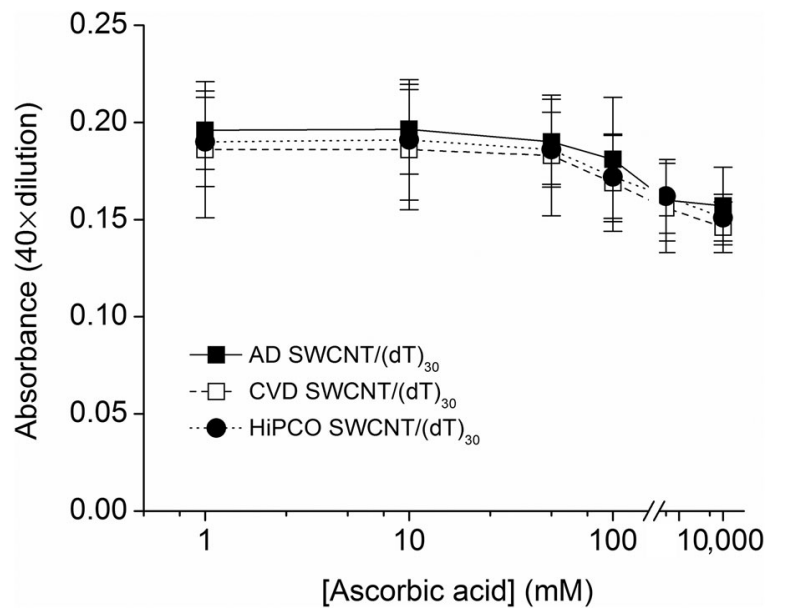

Figure 5. Ascorbic acid concentration-dependent dispersion (absorbance at $1023 \mathrm{~nm}$ ) of $\mathrm{AD}, \mathrm{CVD}$, and HiPCO SWCNTs in the presence of $(\mathrm{dT})_{30}$. Error bars represent SD from three independent repeats of the same experiments. The sonication time is $1 \mathrm{~h}$.

onto the surface of SWCNTs via $\pi$-stacking but not covalent attachment, ${ }^{19,35}$ Hines $^{27}$ reported that dispersion of SWCNTs with $(\mathrm{dT})_{30}$ was achieved through covalent modification of SWCNT surface; free radicals generated during sonication leads to covalent attachment of ssDNA to SWCNT surface, which facilitates the dispersion of SWCNT in an aqueous solution. They showed that SWCNTs were not dispersed with $(\mathrm{dT})_{30}$ in the presence of antioxidants such as ascorbic acid or trolox (chemical structures shown in Figure S1, Supplementary material). ${ }^{27}$ The explanation they provided was that an- tioxidant quenches free radicals during sonication, which prevent covalent functionalization of SWCNTs and covalent attachment of $(\mathrm{dT})_{30}$ to SWCNT surface. To test this possibility, we examined the dispersion of SWCNTs with $(\mathrm{dT})_{30}$ in the presence of either ascorbic acid or trolox. The amount of SWCNTs dispersed into the aqueous solution was measured by UV-Vis absorbance spectra as we showed previously. ${ }^{20}$ As shown in Figure 4, SWCNTs can be dispersed into an aqueous solution in the presence of either antioxidant. The extent of dispersion was quantitatively similar to that without antioxidant (top panels). Moreover, a systematic study on the dependence of SWCNT dispersion on the concentration of ascorbic acid only shows less than $25 \%$ decrease in SWCNT dispersion even at very high concentrations of ascorbic acid (Fig. 5). We further tested the dispersion of SWCNTs in the presence of either antioxidant plus purging with argon to minimize the formation of free radicals during sonication. Throughout, all these different dispersion conditions produced similar extent of SWCNT dispersion, less than $3 \%$ difference was observed in the amount of SWCNTs dispersed under various conditions. This conclusion is true for all three types of SWCNTs that we have tested. Furthermore, we have quantitated the $I_{\mathrm{D}} / I_{\mathrm{G}}$ ratios for all these samples prepared from sonication for $1 \mathrm{~h}$ at $\sim 3 \mathrm{~W}$. As shown in Figure 6, all these samples showed $I_{\mathrm{D}} / I_{\mathrm{G}}$ ratios less than 0.01 , and there were no changes in these values within error in the presence or absence of antioxidant. These results suggest that covalent attachment of $(\mathrm{dT})_{30}$ to the SWCNTs is unlikely to be the mechanism of SWCNT dispersion by $(\mathrm{dT})_{30}$ because no significant differences were observed for SWCNT dispersion in the presence or absence of antioxidants. These results also indicate that under current sonication conditions, the impact of free radical formation on SWCNT dispersion is low. 

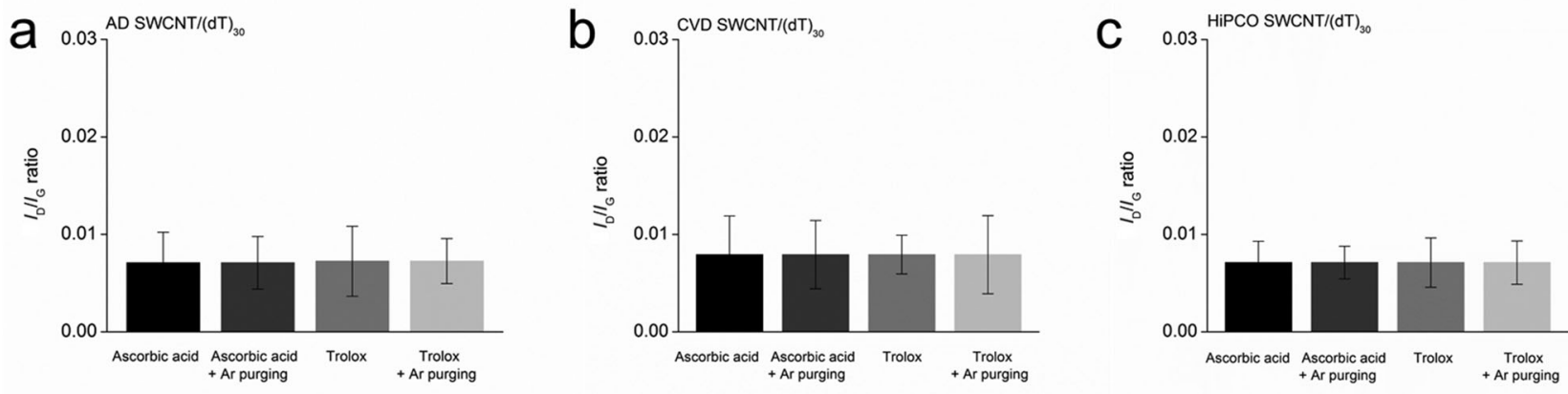

Figure 6. $I_{\mathrm{D}} / I_{\mathrm{G}}$ ratios for (a) $\mathrm{AD}(\mathrm{b}) \mathrm{CVD}$, and (c) HiPCO SWCNT/(dT) $)_{30}$ after $1 \mathrm{~h}$ sonication using a tabletop sonicator $(\sim 3 \mathrm{~W})$ in the presence of ascorbic acid or trolox, with or without argon purging. Error bars represent SD from three independent repeats of the same experiments.

\section{CONCLUSIONS}

Single-walled carbon nanotube dispersion into an aqueous solution using conventional sonication process (bath sonication power: $3-80 \mathrm{~W}$, sonication time: $1-6 \mathrm{~h}$ ) did not induce noticeable covalent modification of SWCNTs compared with SWCNT soot without sonication. Longer sonication time or higher sonication power does lead to more defects on SWCNTs; however, this increase in defect sites is less than $10 \%$ and thus not significant. SWCNT sonication in the presence of antioxidants and with argon purging suggests that blocking free radical formation has no apparent effect on SWCNT dispersion, and that dispersion of SWCNTs by ssDNA is through noncovalent $\pi$-stacking instead of convalent conjugation to SWCNT surface. Overall, our data provide evidence that sonication method during dispersion of SWCNT in an aqueous solution as we described does not induce noticeable surface damage to SWCNTs.

\section{ACKNOWLEDGMENTS}

This work is supported by 3M nontenured faculty grant (W.C.).We thank Dr. Adam Matzger in Department of Chemistry, University of Michigan, for sharing the use of instruments available in his laboratory.

\section{REFERENCES}

1. Liu Z, Fan AC, Rakhra K, Sherlock S, Goodwin A, Chen X, Yang Q, Felsher DW, Dai H. 2009. Supramolecular stacking of doxorubicin on carbon nanotubes for in vivo cancer therapy. Angew Chem Int Ed Engl 48:7668-7672.

2. Liu Z, Tabakman SM, Chen Z, Dai H. 2009. Preparation of carbon nanotube bioconjugates for biomedical applications. Nat Protoc 4:13721382.

3. Siu KS, Chen D, Zheng X, Zhang X, Johnston N, Liu Y, Yuan K, Koropatnick J, Gillies ER, Min WP. 2014. Non-covalently functionalized single-walled carbon nanotube for topical siRNA delivery into melanoma. Biomaterials 35:3435-3442.

4. Sacchetti C, Liu-Bryan R, Magrini A, Rosato N, Bottini N, Bottini M. 2014. Polyethylene-glycol-modified single-walled carbon nanotubes for intra-articular delivery to chondrocytes. ACS Nano 8:12280-12291. 5. Martinelli V, Cellot G, Toma FM, Long CS, Caldwell JH, Zentilin L, Giacca M, Turco A, Prato M, Ballerini L, Mestroni L. 2013. Carbon nanotubes instruct physiological growth and functionally mature syncytia: Nongenetic engineering of cardiac myocytes. ACS Nano 7:5746-5756. 6. Lee HJ, Park J, Yoon OJ, Kim HW, Lee do Y, Kim do H, Lee WB, Lee NE, Bonventre JV, Kim SS. 2011. Amine-modified single-walled carbon nanotubes protect neurons from injury in a rat stroke model. Nat Nanotechnol 6:121-125.

7. Sinani VA, Gheith MK, Yaroslavov AA, Rakhnyanskaya AA, Sun K, Mamedov AA, Wicksted JP, Kotov NA. 2005. Aqueous dispersions of single-wall and multiwall carbon nanotubes with designed amphiphilic polycations. J Am Chem Soc 127:3463-3472.

8. Strano MS, Moore VC, Miller MK, Allen MJ, Haroz EH, Kittrell C, Hauge RH, Smalley RE. 2003. The role of surfactant adsoprtion during ultrasonication in the dispersion of single-walled carbon nanotubes. J Nanosci Nanotechnol 3:81-86.

9. Mutlu GM, Budinger GR, Green AA, Urich D, Soberanes S, Chiarella SE, Alheid GF, McCrimmon DR, Szleifer I, Hersam MC. 2010. Biocompatible nanoscale dispersion of single-walled carbon nanotubes minimizes in vivo pulmonary toxicity. Nano Lett 10:1664-1670.

10. Liu J, Rinzler AG, Dai H, Hafner JH, Bradley RK, Boul PJ, Lu A, Iverson T, Shelimov K, Huffman CB, Rodriguez-Macias F, Shon YS, Lee TR, Colbert DT, Smalley RE. 1998. Fullerene pipes. Science 280:1253-1256.

11. Wang Y, Iqbal Z, Mitra S. 2006. Rapidly functionalized waterdispersed carbon nanotubes at high concentration. J Am Chem Soc 128:95-99.

12. Worsley KA, Kalinina I, Bekyarova E, Haddon RC. 2009. Functionalization and dissolution of nitric acid treated single-walled carbon nanotubes. J Am Chem Soc 131:18153-18158.

13. Furtado CA, Kim UJ, Gutierrez HR, Pan L, Dickey EC, Eklund PC. 2005. Debundling and dissolution of single-walled carbon nanotubes in amide solvents. J Am Chem Soc 126:6095-6105.

14. Liu Z, Tabakman S, Welsher K, Dai H. 2009. Carbon nanotubes in biology and medicine: In vitro and in vivo detection, imaging and drug delivery. Nano Res 2:85-120.

15. Liu Z, Tabakman S, Chen Z, Dai H. 2009. Preparation of carbon nanotube bioconjugates for biomedical applications. Nat Protoc 4:1372_ 1381.

16. Fagan JA, Landi BJ, Mandelbaum I, Simpson JR, Bajpai V, Bauer BJ, Migler K, Walker AR, Raffaelle R, Hobbie EK. 2006. Comparative measures of single-wall carbon nanotube dispersion. J Phys Chem B 110:23801-23805.

17. Simmons TJ, Bult J, Hashim DP, Linhardt RJ, Ajayan PM. 2009. Noncovalent functionalization as an alternative to oxidative acid treatment of single wall carbon nanotubes with applications for polymer composites. ACS Nano 3:865-870.

18. Tu X, Manohar S, Jagota A, Zheng M. 2009. DNA sequence motifs for structure-specific recognition and separation of carbon nanotubes. Nature 460:250-253.

19. Zheng M, Jagota A, Semke ED, Diner BA, Mclean RS, Lustig SR, Richardson RE, Tassi NG. 2003. DNA-assisted dispersion and separation of carbon nanotubes. Nat Mater 2:338-342.

20. Koh B, Park JB, Hou X, Cheng W. 2011. Comparative dispersion studies of single-walled carbon nanotubes in aqueous solution. J Phys Chem B 115:2627-2633. 
21. Koh B, Kim G, Yoon HK, Park JB, Kopelman R, Cheng W. 2012. Fluorophore and dye-assisted dispersion of carbon nanotubes in aqueous solution. Langmuir 28:11676-11686.

22. Chen RJ, Bangsaruntip S, Drouvalakis KA, Kam NWS, Shim M, Li Y, Kim W, Utz PJ, Dai H. 2003. Noncovalent fuctionalization of carbon nanotubes for highly specific electronic biosensors. Proc Natl Acad Sci USA 100:4984-4989.

23. Murakami H, Nomura T, Nakashima N. 2003. Noncovalent porphyrin-functionalized single-walled carbon nanotubes in solution and the formation of porphyrin-nanotube nanocomposites. Chem Phys Lett 378:481-485.

24. Huang W, Lin Y, Taylor S, Gaillard J, Mao AM, Sun YP. 2002. Sonication-assisted functionalization and solubilization of carbon nanotubes. Nano Lett 2:231-234.

25. Riesz P, Berdahl D, Christman CL. 1985. Free radical generation by ultrasound in aqueous and nonaqueous solutions. Environ Health Perspect 64:233-252.

26. Elia P, Azoulay A, Zeiri Y. 2012. On the efficiency of water soluble antioxidants. Ultrason Sonochem 19:314-324.

27. Hines BDD. 2009. Sonication-mediated association of DNA with single-walled carbon nanotubes: Characterization and potential biological applications. Ph.D. Thesis. West Lafayette, Indiana: Purdue University.

28. Hennrich F, Krupke R, Lebedkin S, Arnold K, Fischer R, Resasco DE, Kappes MM. 2005. Raman spectroscopy of individual single-walled carbon nanotubes. J Phys Chem B 109:10567-10573.
29. Cancado LG, Jorio A, Martins Ferreira EH, Stavale F, Achete CA, Capaz RB, Moutinho MVO, Lombardo A, Kulmala TS, Ferrari AC. 2011. Quantifying defects in graphene via Raman spectroscopy at different excitation energies. Nano Lett 11:31903196.

30. Freiman S, Hooker S, Migler K, Arepalli S. 2008. Measurement issues in single walled carbon nanotubes. Gaithersburg, Maryland: NIST Special Publication, pp 960-919.

31. Chou SG, Son H, Kong J, Jorio A, Saito R, Zheng M, Dresselhaus G, Dresselhaus MS. 2007. Length characterization of DNA-wrapped carbon nanotubes using Raman spectroscopy. Appl Phys Lett 90: 131109 .

32. Hof A, Bosch S, Englert JM, Hauke F, Hirsch A. 2012. Statistical Raman spectroscopy: An method for the characterization of covalently functionalized single-walled carbon nanotubes. Angew Chem Int Ed Engl 51:11727-11730.

33. Niki E. 1991. Action of ascorbic acid as a scavenger of active and stable oxygen radicals. Am J Clin Nutr 54:1119-1124.

34. Bagchi D, Garg A, Krohn RL, Bagchi M, Tran MX, Stohs SJ. 1997. Oxygen free radical scavenging abilities of vitamins $\mathrm{C}$ and $\mathrm{E}$, and a grape seed proanthocyanidin extract in vitro. Res Commun Mol Pathol Pharmacol 95:179-189.

35. Campbell JF, Tessmer I, Holden Thorp H, Erie DE. 2008. Atomic force microscopy studies of DNA-wrapped carbon nanotube structure and binding to quantum dots. J Am Chem Soc 130:1064810655 . 